\title{
Contrast Echocardiography and Migraine in Divers with Patent Foramen Ovale
}

\author{
Roberto Di Fabio, Elisabetta Giugni, Imerio Angeloni, Nicola Vanacore, Carlo \\ Casali, Alberto Pierallini, Rita Vadalà, Francesco Pierelli
}

\begin{abstract}
Background: It has been proposed that the patent foramen ovale (PFO) may be associated with migraine, in particular migraine with aura. However, it is not clear whether paradoxical embolism triggers crises of headache. Cerebral embolization is provoked in subjects with PFO through contrast echocardiography, a safe method to diagnose the presence of foramen ovale pervium. Methods: Twenty-four men practicing diving, an activity characterized by increased prevalence of PFO and migraine, underwent trans-thoracic echocardiography with contrast solution, composed of saline and air mixture and checked for the occurrence of migraine in the following 24 hours. Results: A PFO (five of minimal size, i.e. visible only during Valsalva, one of small and two of medium size) was detected in $8 / 24$ divers $(33 \%)$. No one reported headache over the 24 hours after the procedure. Discussion: Our preliminary data suggest that cerebral micro-embolism, provoked by contrast echocardiography, does not systematically trigger migraine crises when a minimal-to-medium sized patent foramen ovale is present.
\end{abstract}

RÉSUMÉ: Échocardiographie de contraste et migraine chez les plongeurs porteurs d'un foramen ovale perméable. Contexte : Selon certains, un foramen ovale perméable (FOP) pourrait être associé à la migraine, particulièrement à la migraine avec aura. Cependant, on ne sait pas si les crises de céphalée sont déclenchées par embolie paroxystique. L'embolie cérébrale a été provoquée chez des sujets porteurs d'un FOP au moyen de l'échocardiographie de contraste, une méthode sûre pour diagnostiquer la présence d'un FOP. Méthodes : Vingtquatre hommes adeptes de la plongée, une activité caractérisée par une prévalence augmentée de FOP et de migraine, ont subi un échocardiogramme transthoracique avec un mélange de salin et d'air comme solution de contraste. Ils ont été suivis pendant 24 heures pour vérifier l'apparition de migraine. Résultats : Des FOP, dont 5 de taille minime c'est-à-dire visibles seulement pendant une manœuvre de Valsalva, un petit et deux de taille moyenne, ont été détectés chez 8 des 24 plongeurs (33\%). Personne n'a rapporté avoir souffert de céphalée au cours des 24 heures suivant l'examen. Discussion : Selon nos données préliminaires, la microembolisation cérébrale provoquée par l'échocardiographie de contraste ne déclenche pas systématiquement des crises de migraine en présence d'un FOP de taille minime à moyenne.

Can. J. Neurol. Sci. 2009; 36: 740-744

The causes of migraine are still unknown, but several triggers of the attacks have been identified so far. ${ }^{1}$

It has been hypothesized that the presence of patent foramen ovale (PFO) and of paradoxical cerebral micro-embolism may increase the risk of migraine, ${ }^{2}$ in particular migraine with aura. ${ }^{3,4}$ In facts, patients suffering from migraine (with aura and without aura) showed higher prevalence of PFO. ${ }^{4-6}$

Moreover, the trans-catheter closure of cardiac shunts showed a certain effect on the frequency and severity of attacks in patients with migraine. However, when an incomplete closure can be detected by contrast echocardiography, i.e. echo- cardiography and peripheral injection of an air-water solution, the residual passage of micro-bubbles from the right to the left

From the Department of Neuromotor Rehabilitation (RDF, CC, FP), "Sapienza" University of Rome Polo Pontino - I.C.O.T., Latina; Department of Radiology (EG, AP, RV), IRCSS-S Raffaele Pisana; Cardiological Unit (IA), ASL RME; Department of Epidemiology (NV), National Health Institute, Rome; IRCCS-Neuromed (FP), Pozzilli, Italy.

Received May 19, 2009. Final Revisions Submitted June 15, 2009. Correspondence to: Roberto Di Fabio, Department of Neuromotor Rehabilitation, "Sapienza" University of Rome Polo Pontino - I.C.O.T., via Francesco Faggiana 34, 004100, Latina, Italy.E-mail: rob.dif@tiscali.it 
circulation during the diagnostic procedure is sufficient to induce migraine crises. ${ }^{7}$

This supports the idea that micro-bubbles entering the brain circulation can trigger a headache, as also shown in rats, in which many cortical stimuli including ischemia can induce cortical spreading depression, the putative electrophysiological event underlying migraine. ${ }^{8}$

Diving, an activity characterized by high intra-thoracic pressures and frequent Valsalva manoeuvres which are performed to re-equilibrate the pressure in the middle ear, is accompanied by high risk of paradoxical brain micro-embolism and migraine. .-12 $^{-12}$

It has been shown that divers with PFO suffer from migraine more frequently than divers without PFO. In particular, larger PFO are associated with increased prevalence of migraine with aura, both after diving and in everyday life. ${ }^{13}$

Contrast trans-thoracic echocardiography is a safe and simple procedure to assess the presence of a foramen ovale pervium. The contrast, an agitated air-saline solution, causes a harmless pulmonary micro-embolism. When a PFO is present, the inoculation of the solution in a peripheral vein may cause an apparently innocuous cerebral micro-embolism.

To establish whether cerebral micro-embolism may initiate migraine crises in PFO carriers we studied 24 divers, using a contrast trans-thoracic echocardiographic technique.
If cerebral micro-embolism represents a trigger for migraine, we should expect that in divers with PFO, rather than in divers without PFO, the inoculation of the contrast may induce migraine attacks during or after the echocardiographic procedure.

\section{METHODS}

Study population. Twenty-four professional male scuba divers participated in this study. The subjects are part of the Emergency firefighter scuba unit of Rome, throughout the Italian region of Lazio, working in safety operations. The enrolment was based on volunteer participation.

All the participants were given a complete description of the study and gave their informed consent to take part. The local ethics committee approved the study.

Women were not included, to avoid an additional variable represented by hormonal therapy or menses, which may conceal the occurrence of migraine.

Divers use compressed air and limited their descent to 50 metres depth (see Table 1).

The subjects were interviewed carefully for their diving and medical history, including medication, alcohol use and smoking habits. No subject had a history of stroke or transient ischemic attack. The blood screening, including hemochrome, glycaemia, liver and kidney functions, protein electrophoresis, blood

Table 1: General characteristics of a population of divers $(n=24)$

\begin{tabular}{|c|c|c|c|}
\hline \multicolumn{2}{|l|}{ Age, years } & \multicolumn{2}{|c|}{$40.1 \pm 6.4($ range $29-53)$} \\
\hline \multirow[t]{4}{*}{ Education,n (\%) } & Elementary school & $1(4)$ & \\
\hline & Intermediate school & $19(79)$ & \\
\hline & High school & $2(8)$ & \\
\hline & Degree & $2(8)$ & \\
\hline \multicolumn{2}{|l|}{ Body Mass Index } & $26.6 \pm 4.2$ & \\
\hline \multicolumn{2}{|l|}{ Hypertension,n (\%) } & $1(4)$ & \\
\hline \multirow[t]{3}{*}{ Smoking,n (\%) } & Never & $14(58)$ & \\
\hline & $<3$ cigarette/day & $10(42)$ & \\
\hline & $>3$ cigarette/day & $0(0)$ & \\
\hline \multirow[t]{3}{*}{ Alcohol use,n (\%) } & None & $12(50)$ & \\
\hline & $<200 \mathrm{ml} /$ day & $12(50)$ & \\
\hline & $>200 \mathrm{ml} /$ day & 0 & \\
\hline \multicolumn{2}{|l|}{ Diabetes, $n$} & 0 & \\
\hline \multicolumn{2}{|l|}{ Drugs,n } & $1(4)$ & $\begin{array}{l}\text { one diver assumes furosemide per os }(10 \mathrm{mg} / 3 \text { times a } \\
\text { week) }\end{array}$ \\
\hline \multicolumn{2}{|c|}{ Coagulation abnormalities, $\mathrm{n}$} & 0 & \\
\hline \multicolumn{2}{|l|}{ High cholesterol,n } & 0 & \\
\hline \multicolumn{2}{|c|}{ Tendon hyper-reflexia } & 0 & \\
\hline \multicolumn{2}{|c|}{ Scuba dives per year (max $50 \mathrm{mt}$ ) } & $467 \pm 441$ & 40-1950) \\
\hline \multicolumn{2}{|l|}{ Migraine,n (\%) } & $6(25)$ & \\
\hline & MoA & $5(20.8)$ & one crisis/week $(n=3)$, one crisis/month $(n=2)$ \\
\hline & MA & $1 \quad(4.2)$ & one crisis $>$ three months \\
\hline \multicolumn{2}{|c|}{ Tension type headache,n (\%) } & $9(37.5)$ & \\
\hline \multicolumn{2}{|c|}{ Decompression Sickness,n (\%) } & $3(12.5)$ & \\
\hline
\end{tabular}

$\mathrm{MoA}=$ migraine without aura; $\mathrm{MA}=$ migraine with aura; $\mathrm{n}=$ number 
Table 2: Characteristics of divers without and with PFO

\begin{tabular}{lccc}
\hline & Divers without PFO (n=16) & $\begin{array}{c}\text { Divers with } \\
\text { PFO } \\
(\mathbf{n}=\mathbf{8})\end{array}$ & P \\
\hline mean \pm SD & & $\mathrm{ns}$ \\
\hline Age, years & $38.9 \pm 5.5$ & $44.5 \pm 6.9$ & $\mathrm{~ns}$ \\
Dives, $\mathrm{n}$ & $447.5 \pm 486.3$ & $513.9 \pm 359.2$ & $\mathrm{~ns}$ \\
Bio-mass Index & $26.6 \pm 4.7$ & $26.4 \pm 3.0$ & $\mathrm{~ns}$ \\
Education level, years & $2.3 \pm 0.7$ & $1.9 \pm 0.4$ & $\mathrm{~ns}$ \\
Height, cm & $177.9 \pm 6.1$ & $173.7 \pm 4.9$ & $\mathrm{~ns}$ \\
Weight, kg & $82.0 \pm 11.6$ & $79.6 \pm 10.1$ & $\mathrm{~ns}$ \\
Cigarettes, n & $1.5 \pm 2.3$ & $1.9 \pm 2.6$ & $\mathrm{~ns}$ \\
Brain lesions, $\mathrm{n}$ & $1.3 \pm 2.4$ & $10.8 \pm 26.5$ & \\
\hline
\end{tabular}

$\mathrm{n}=$ number; $\mathrm{ns}=$ not significant

coagulation, cholesterol and lipoprotein dosage, and the blood pressure at rest were normal. Body-mass index (BMI) was calculated as weight $(\mathrm{Kg}) /$ height $(\mathrm{mt})^{2}$. All subjects underwent a normal electrocardiogram stress test, performed in a separate session.

An otolaryngologic evaluation, including audiological and vestibular assessment excluded any malfunction of the middle or inner ear. No diver showed any abnormality at the neurological examination.

All subjects underwent brain magnetic resonance investigation (Gyroscan NT Intera Philips 1.5 Tesla), and cerebral ischemic lesions were defined as areas of abnormally high signal intensity (hyper-intense lesions) on DP-T2 and FLAIR sequences, according to the article of Vermeer et al. ${ }^{14}$

Divers were interviewed about major decompression sickness episodes (DCS). This syndrome, frequently observed in PFO carriers includes a wide spectrum of neurological signs, such as paralyses, paresthesias, vertigo, ataxia, speech disturbances, unconsciousness, cognitive and visual deficits, urinary incontinence, as well as headache. ${ }^{15,16}$ Guidelines for the diagnosis of migraine, according to the International Classification of Headache Disorders, 2nd edition, were applied in the study group. ${ }^{17}$

A questionnaire about migraine was completed after the echocardiography, with divers unaware of the results of the cardiologic procedure. Blood pressure measured after the echocardiogram was normal.

The results of the magnetic resonance and echocardiography were given directly to the participants.
Trans-thoracic Echocardiography investigation. It was performed with an imaging system Acuson Sequoia using a multi-hertz probe and second harmonic function. The images were acquired from apical, para-sternal and sub-costal views. A patent foramen ovale was defined as the occurance of at least one was detected in the left atrium within three cycles after peripheral injection of contrast agent into the ante-cubital vein (agitated saline: $9 \mathrm{ml}$ of saline solution plus $1 \mathrm{cc}$ of air). The test was repeated twice at rest and twice during ten seconds of Valsalva manoeuvre, performed three seconds after the saline injection. The shunt was considered small when less than ten micro-bubbles were detected, medium if more than ten micro-bubbles were seen and large if all the left atrium was opaque after contrast injection. Regarding the classification used in the article of Souteyrand et al, we established the "minimal" grade of PFO as if any microbubble was detected only with Valsalva manoeuvre but not at rest. ${ }^{18}$

Statistical analysis. We used the unpaired two-tailed Student's t-test to compare normally distributed variables

(age, BMI, height, weight, number of dives per year, number of cigarettes per day, education level) between the two groups (PFO/no PFO carriers).

The Statistical Package for the Social Sciences (SPSS) for Windows, version 16.0 was used for the analyses.

\section{RESUlts}

The general characteristics of the participants are shown in Table 1.

In their medical history, six out of 24 (25\%) divers suffered from migraine, one diagnosed as migraine with aura. Among the six migraineurs, one diver showed a small PFO and another diver had a medium-sized PFO. This latter suffered from migraine with aura, which consisted of rare episodes of paresthesias on the left side of the body.

In all subjects, the history of migraine predated the diving career.

The migraineurs reported a crisis once a week $(n=3)$ or once a month $(n=3)$. Nine divers $(37.5 \%)$ complained of episodic tension headache (one to two crises per month).

The intensity of the migraine crises was generally described as low, and rarely divers reported nausea, photo- or phonophobia. The six migraineurs complained of worsening of the symptoms with physical exertion. Only one diver reported a migraine crisis soon after diving.

Eight PFO were detected in 24 divers (33\%). In five subjects, the dimension of PFO was minimal, i.e. visible only during Valsalva. In one diver, PFO was of small entity, and in two, a medium foramen ovale was found. No large foramen ovale was recognized in this study group. 
Twelve out of 24 divers (50\%) showed brain spots on MR. Seven divers $(29.1 \%)$ had multiple cerebral foci $(>2)$. The majority of the foci were detected in the frontal and temporal regions, mostly consisting of spots $<5 \mathrm{~mm}$ in the deep white matter.

The divers with PFO were similar to the divers without PFO, with respect to the age, BMI, height, weight, dives performed per year, education level, and smoking habits.

The presence of PFO was not associated with higher prevalence of migraine, DCS or brain lesions (Table 2).

No diver suffered from any headache crisis soon after or in the 24 hours following the echocardiographic exam. Only one diver reported nausea, without migraine, starting after the echocardiography. The following day, he suffered from flu-like symptoms. This diver did not usually have migraine.

\section{Discussion}

Several elements, such as physical exertion, stress, intense lights, noises, odours or a variety of foods are capable of provoking an attack of migraine, although the relationship between these triggers and the crises is not always obvious. ${ }^{1,19}$

Diving, an activity associated with several conditions which promote the haematic formation of air micro-bubbles, can favour the passage of micro-emboli to the brain circulation through a right-to-left shunt. ${ }^{9-11,13}$

In divers, cerebral embolism caused by nitrogen bubbles entering the brain circulation leads to decompression sickness, a neurological disease often including headache. ${ }^{13,15,16}$

Divers suffer commonly from migraine and show a high prevalence of PFO. ${ }^{15,20}$ Wilmshurst et al described 37 patients who improved the severity and the frequency of migraine attacks after PFO trans-catheter closure. Two of them developed migraine during the contrast echocardiogram performed six weeks and six months after the closure procedure. ${ }^{13}$

Therefore, we decided to investigate whether direct cerebral micro-embolism, provoked during contrast echocardiography, represents a trigger for migraine in a group of professional divers, who are usually exposed to increased environmental pressures and perform the Valsalva manoeuvre routinely.

Migraine, characterized by infrequent and weak crises, was found in $25 \%$ of divers studied. Although there are no casecontrol studies investigating the prevalence of migraine in divers with respect to a normal population, the particular stressful conditions such as, for instance, increased environmental pressures, immersions in cold water, the rocking of the boat, intense solar brightness, and physical exertion, that divers have to deal with, may account for the common diagnosis of migraine in these subjects. ${ }^{15}$

The patent foramen pervium was evident in $33 \%$ of our study group, resembling the prevalence in the Caucasian population, ${ }^{21}$ but no large foramen was detected.

We did not induce any migraine crisis in the divers with PFO when we injected the contrast solution and in the 24 hours following the cardiologic protocol. One subject, who had a medium PFO, developed nausea, without migraine, after the echocardiography, and the following day he was suffering from flu. This diver did not usually complain of migraine.

Subjects with migraine with aura may experience a migraine attack during contrast echocardiography, performed after the surgical closure of PFO, even if the residual communication is minimal. ${ }^{13}$ Whilst other data in the literature support the hypothesis that brain micro-embolism can be linked to migraine, ${ }^{2-5}$ the contrast protocol that we used to assess the presence of PFO did not trigger any migraine crisis in our divers. This result can be explained by hypothesizing that small brain micro-embolization is not sufficient to induce migraine crises or it can trigger migraine only if a previous sensitization has been produced, as in carriers of large PFO who have experienced migraine with aura for many years..$^{5,7,13}$

If we embrace the current hypothesis proposed by several authors ${ }^{4,11-13,20}$ that the PFO size increases the risk of paradoxical brain embolism, i.e. only larger PFO are at high risk, we are not surprised by the absence of significant differences in the prevalence of migraine, cerebral lesions and decompression sickness episodes between our group of divers with PFO and our group of divers without PFO. Major DCS episodes requiring an hyper-baric treatment occurred in only three subjects (segmental paresis in two subjects and dysarthria in one), who did not show patency of foramen ovale. Moreover, the striking difference in the number of brain lesions between non-carriers and carriers of PFO, as evident in Table 2, was due to one diver who had a medium PFO and 76 lesions. When he was excluded from the statistical analysis, the amount of brain lesions was similar in the groups (divers without PFO vs divers with PFO: $1.3 \pm 2.4$ vs 1.5 $\pm 3.7 P=\mathrm{ns}$ ).

Furthermore, migraineurs did not show an increased prevalence of PFO, brain lesions or DCS in our study population.

The major concern about our study is the small sample size, which makes our results exploratory, but not definitive. A more definitive research should study a broader population, which includes subjects with larger PFO, although the use of contrast in these subjects may not be completely devoid of risks. ${ }^{13}$ Moreover, although contrast echocardiogram showed good accuracy in detecting the presence of right-to-left shunts, contrast trans-cranial Doppler would be more appropriate in assessing the actual amount of micro-bubbles reaching the brain circulation. ${ }^{18}$

Although bubbles have been clearly identified in the left cardiac circulation of PFO carriers during echocardiography, it is not possible to assess the real amount of brain emboli during diving.

In conclusion, our preliminary findings support the hypothesis that cerebral micro-embolism is not a reliable mechanism capable of provoking migraine crises when a minimal-to-medium patent foramen ovale is present. It would be interesting to test our protocol in several cases of larger PFO when massive cerebral micro-embolism is supposed.

\section{ACKNOWLEDGEMENTS}

The authors thank Roberto Proietti who contributed to the organization of the divers schedules and the subjects who voluntarily participated in this study. 


\section{REFERENCES}

1. Lambert GA, Zagami AS. The mode of action of migraine triggers: a hypothesis. Headache. 2009 Feb;49(2):253-75.

2. Anzola GP, Morandi E, Casilli F, Onorato E. Different degrees of right-to-left shunting predict migraine and stroke: data from 420 patients. Neurology. 2006 Mar 14;66(5):765-7.

3. Wilmshurst P, Nightingale S, Pearson M, Morrison L, Walsh K. Relation of atrial shunts to migraine in patients with ischemic stroke and peripheral emboli. Am J Cardiol. 2006 Sep 15;98(6): 831-3.

4. Schwedt TJ, Demaerschalk BM, Dodick DW. Patent foramen ovale and migraine: a quantitative systematic review. 2008 May;28(5): 531-40.

5. Anzola GP, Magoni M, Guindani M, Rozzini L, Dalla Volta G. Potential source of cerebral embolism in migraine with aura. A transcranial Doppler study. Neurology. 1999 May 12;52(8): 1622-5.

6. Dalla Volta G, Guindani M, Zavarise P, Griffini S, Pezzini A, Padovani A. Prevalence of patent foramen ovale in a large series of patients with migraine with aura, migraine without aura and cluster headache, and relationship with clinical phenotype. J Headache Pain. 2005 Sep;6(4):328-30.

7. Wilmshurst PT, Nightingale S, Walsh KP, Morrison WL. Effect on migraine of closure of cardiac right-to-left shunts to prevent recurrence of decompression illness or stroke or for haemodynamic reasons. Lancet. 2000 Nov 11;356(9242): 1648-51.

8. Röther J, De Crespigny AJ, D'Arceuil H, Moseley ME. MR detection of cortical spreading depression immediately after focal ischemia in the rat. J Cereb Blood Flow Metab. 1996 Mar; 16(2):214-20.

9. Reul J, Weis J, Jung A, Willmes K, Thron A. Central nervous system lesions and cervical disc herniations in amateur divers. Lancet. 1995 Jun 3;345(8962):1403-5.

10. Knauth M, Ries S, Pohimann S, Kerby T, Forsting M, Daffertshofer $\mathrm{M}$, et al. Cohort study of multiple brain lesions in sport divers: role of a patent foramen ovale. BMJ. 1997 Mar 8;314(7082): 701-5.
11. Schwerzmann M, Seiler C, Lipp E, Guzman R, Lövblad KO, Kraus $\mathrm{M}$, et al. Relation between directly detected patent foramen ovale and ischemic brain lesions in sport divers. Ann Intern Med. 2001 Jan 2;134(1):21-4.

12. Torti SR, Billinger M, Schwerzmann M, Vogel R, Zbinden R, Windecker S, et al. Risk of decompression illness among 230 divers in relation to the presence and size of patent foramen ovale. Eur Heart J. 2004 Jun;25(12):1014-20.

13. Wilmshurst P, Nightingale S. Relationship between migraine and cardiac and pulmonary right-to-left shunts. Clin Sci (Lond). $2001 \mathrm{Feb} ; 100(2): 215-20$.

14. Vermeer SE, Longstreth WT Jr, Koudstaal PJ. Silent brain infarcts: a systematic review. Lancet Neurol. 2007 Jul;6(7):611-9.

15. Cheshire WP, Ott MC. Headache in divers. Headache. 2001 Mar;41 (3):235-47.

16. Elliott DH, Moon RE. Long-term health effects of diving. In: Bennett PB, Elliott DH, editors. The physiology and medicine of diving. Philadelphia: WB Saunders; 1993. p. 585-604.

17. The International Classification of Headache Disorders. 2nd ed. Cephalalgia. 2004;24 Suppl 1:9-160.

18. Souteyrand G, Motreff P, Lusson JR, Rodriguez R, Geoffroy E, Dauphin $\mathrm{C}$, et al. Comparison of transthoracic echocardiography using second harmonic imaging, transcranial Doppler and transesophageal echocardiography for the detection of patent foramen ovale in stroke patients. Eur J Echocardiogr. 2006 Mar; 7(2):147-54.

19. Arulmani U, Gupta S, Van Den Brink AM, Centurión D, Villalón CM, Saxena PR. Experimental migraine models and their relevance in migraine therapy. Cephalalgia. 2006 Jun;26(6): 642-59.

20. Germonpre P, Hastir F, Dendale P, Marroni A, Nguyen AF, Balestra C. Evidence for increasing patency of the formane ovale in divers. Am J Cardiol. 2005 Apr 1;95(7):912-5.

21. Hagen PT, Scholz DG, Edwards WD. Incidence and size of patent foramen ovale during the first ten decades of life: an autopsy study of 965 normal hearts. Mayo Clin Proc. 1984 Jan;59(1): $17-20$. 